\title{
Orientierungslos, mutlos, machtlos
}

\section{Die Idee der Bürgergesellschaft hat es noch nicht zum gesellschaftlichen Leitbild geschafft - Das liegt auch an uns selbst}

Stefan Nährlich

Die Idee der Bürgergesellschaft wäre gescheitert, käme sie über bloßes staatsergänzendes und staatsentlastendes Handeln nicht hinaus. Denn sie ist mehr als die Addition von Spendensummen, ehrenamtlicher Arbeit und gemeinnützigen $\mathrm{O} r$ ganisationen. Analog zum marktlichen Steuerungsideal der Konsumentensouveränität, wäre die Orientierung an einer Bürgersouveränität als letztgültigem "Regler " gesellschaftlicher Prozesse, das Steuerungsideal für eine auf Selbstorganisation basierende Bürgergesellschaft.

Zum großen Gesellschaftsentwurf ist sie noch nicht geworden, die Idee der Bürgergesellschaft. $\mathrm{Zu}$ eingefahren sind die Institutionen und Denkroutinen in Deutschland, wo Gesellschaftspolitik vornehmlich in Kategorien staatlichen Handelns gedacht wird: vom Sozialstaat über den »aktivierenden Staat « bis zum »vorsorgenden Staat « - Hauptsache Staat, auch wenn die Qualität immer geringer wird. Auf der anderen Seite kommt die Idee der sozialen Marktwirtschaft nicht über das Attribut einer neuen sozialen Marktwirtschaft hinaus, als ob die nationale Volkswirtschaft der Bundesrepublik in Zeiten der Globalisierung einfach irgendwie weiterbestünde.

Dabei ist die von allen Seiten und nahezu im Konsens postulierte größere Eigenverantwortung der Bürger das Eingeständnis von dem Erreichen und Überschreiten der Grenzen dessen, was an administrativer Steuer- und Planbarkeit in komplexen gesellschaftlichen und wirt-

Dr. Stefan Nährlich ist Geschäftsführer der Aktiven Bürgerschaft, dem

Kompetenzzentrum für Bürgerengagement der Volksbanken und Raiffeisenbanken (vgl. Autorenporträt Seite 152).

E-Mail

stefan.naehrlich@aktive-buergerschaft.de schaftlichen Zusammenhängen möglich ist. Gleichzeitig erweist sich der Marktmechanismus allein als nicht ausreichend, um Antworten auf Fragen wie Gerechtigkeit, gesellschaftliche Teilhabe oder Solidarität zu finden. Mehr Eigenverantwortung ist daher der erste und richtige Schritt zu mehr Selbstorganisation, und mehr Selbstorganisation ist der einzige Weg, um komplexe Zusammenhänge zu bewältigen und gleichzeitig individuelle Freiheit und gesellschaftliche Dynamik wieder voll zu entfalten.

Auf zwei Tagungen in Berlin, dem Fachkongress »Bürgergesellschaft Wunsch und Wirklichkeit « im Wissenschaftszentrum Berlin im Oktober letzten Jahres und dem Hauptstadtkongress-Forum »Die Berliner Bürgergesellschaft organisiert sich: Berlin hilft sich selbst! «im Januar diesen Jahres, fielen drei Probleme der bürgergesellschaftlichen Avantgarde besonders auf:

1. Orientierungslos: Bürgerschaftliches -Engagement hat nicht nur in der öffentlichen und veröffentlichten Meinung sondern auch in der konkreten Praxis eine durchaus bemerkenswerte Entwicklung genommen. Vom »neuen Ehrenamt « über die »Freiwilligenarbeit « bis zum »bürgerschaftlichen Engagement « ist der Begriff konsequent und richtigerweise ausgedehnt worden. Jenseits des klassischen Ehrenamtes, das selbst vielfach organisationsinternen Modernisierungsprozessen ausgesetzt ist, sind neue Engagementformen wie Freiwilligenagenturen oder Bürgerstiftungen entstanden. Das Stiftungswesen boomt derart, dass die Hälfte aller bestehenden Stiftungen in Deutschland Neugründungen der letzten 15 Jahre sind, mithin jünger als das wiedervereinigte Deutschland.

Auf der konzeptionellen Ebene ist die Bürgergesellschaft jedoch nach wie vor eine Idee ohne Theorie, wie Warnfried Dettling richtig schrieb. Nichts ist so praktisch wie eine gute Theorie, denn sie reduziert Komplexität und gibt Orientierung für konsistentes und zielgerichtetes Handeln, sowohl ordnungspolitisch als auch im Alltagsleben. Jürgen Kocka, bis vor kurzem Präsident des Wissenschaftszentrums Berlin für Sozialforschung, hat darauf hingewiesen. Zentral für die Bürgergesellschaft ist gesellschaftliche Selbstorganisation, etwa durch Vereine, Zirkel oder Netzwerke. Analog zum marktlichen Steuerungsideal der Konsumentensouveränität, wäre die Orientierung an einer Bürgersouveränität als letztgültigem »Regler « gesellschaftlicher Prozesse, das Steuerungsideal für eine auf Selbstorganisation basierende Bürgergesellschaft.

Die Idee der Bürgergesellschaft wäre gescheitert, käme sie über bloßes staatsergänzendes und staatsentlastendes Handeln nicht hinaus. Denn sie ist mehr als die Addition von Spendensummen, ehrenamtlicher Arbeit und gemeinnützigen Organisationen. Gleichwohl ist der Begriffskern von Bürgergesellschaft schwer zu greifen, seine konzeptionellen Merkmale sind bislang offenbar kaum angemessen medial zu vermitteln gewesen, so dass man jüngst unverhofft in der Debatte über die »neue Bürgerlichkeit « landete.

2 Mutlos: Nach dem Schlagwort von •der »Anerkennungskultur «, ist der Wunsch »sich auf gleicher Augenhöhe zu begegnen « eine der immer wiederkehrenden Forderungen in einschlägigen Reden oder Beiträgen von Vertretern bürgerschaftlichen Engagements. Worin wurzelt eigentlich dieser offenbar von vielen empfundene Mangel an Respekt? Dass bürgerschaftlich Engagierte und gemeinnützige Organisationen als Zuwendungsempfänger der öffentlichen Hand meist in einer Bittstellerposition sind, ist zwar richtig, und insofern mag das Sein auch das Bewusstsein bestimmen, aber dies ist wohl nur die halbe Wahrheit. Schließlich ist die Wirtschaft der größte Subventionsempfänger und streicht den Löwenanteil der im Jahr 2005 gezahlten Subventionen 
in Höhe von 55,6 Milliarden Euro (Subventionsbericht der Bundesregierung) oder 145 Milliarden Euro (Angaben des Kieler Instituts für Weltwirtschaft) ein, ohne dass dies erkennbare Selbstzweifel bei Managern, Unternehmern oder Verbandsfunktionären mit sich brächte. Die andere Hälfte der Wahrheit ist nämlich, dass sich die Wirtschaft über ihre Leistungsfähigkeit und Leistung für die Gesellschaft im Klaren ist, wenn auch letzteres aktuell in der Kritik steht. Auch ein »vaterlandsloser « Exportweltmeister, um hier die Kritik von Ex-Kanzler Schröder an der Arbeitsplatzverlagerung deutscher Unternehmen in Ausland anzuführen, bleibt ein Weltmeister.

Was hingegen leistet bürgerschaftliches Engagement, worauf sich das Selbstbewusstsein begründen könnte? Dass Ehren- und Hauptamtliche, Vereine und Stiftungen einen wichtigen Beitrag leisten, davon sind die meisten überzeugt und haben auch eine Vorstellung davon, worin dieser Beitrag besteht. In der Produktion von »Sozialkapital «, das gut für die Leistungsfähigkeit der Gesellschaft ist, im »sozialen Kitt «, der unsere Gesellschaft zusammenhält oder in den "vielen Dingen, die es ohne ehrenamtliches Engagement nicht mehr gäbe «. Das alles ist plausibel, aber ob und unter welchen Umständen das konkret so ist, welche Ergebnisse und Folgen die Arbeit von engagierten Bürgern und gemeinnützigen Organisationen hat, bleibt offen. Bei der Aufrechterhaltung dieses nebulösen $\mathrm{Zu}$ standes sind sich Zuwendungsgeber und Zuwendungsnehmer nicht selten einig, wie man beispielhaft bei der Evaluierung der Maßnahmen und Programme gegen Fremdenfeindlichkeit (»Aufstand der Anständigen «) erleben konnte.

3. Machtlos: Dass bürgerschaftliches -Engagement die Machtfrage aufwirft, ist eigentlich nicht neu. Doch jenseits üblicher Beharrungskräfte, die es bei Veränderungen immer und überall gibt, besteht in dem Zusammenwirken von Politik und Bürgerengagement oft- befriedigend und frustrierend empfunden wird und in seiner Wirkung eher engagementhemmend wirkt, ist beispielsweise aus der Begleitforschung zu formellen Beteiligungsverfahren gut bekannt. Das Problem der Machtfrage liegt aber wohlmöglich nicht nur in der postulierten Unfähigkeit der Politik, Macht nicht abgeben zu können, sondern auch in der mangelnden Bereitschaft bürgerschaftlichen Engagements, Verantwortung übernehmen zu wollen.

\section{"Mehr Selbstorganisation scheint der einzige Weg, um sowohl komplexe Zusammenhänge zu bewältigen als auch individuelle Freibeit zu sichern «}

mals ein Problem: Die Machtfrage wird nicht konsequent genug gestellt. Politiker machen zu Recht geltend, dass Sie für Entscheidungen in ihrem Zuständigkeitsbereich die Verantwortung tragen, mithin auch das Risiko, dafür vom Wähler (oder innerparteilich) »abgestraft « zu werden. Konsequenterweise wollen sie daher in der Zusammenarbeit mit bürgerschaftlich Engagierten das letzte Wort haben, was wie getan - oder eben nicht getan wird. Bürgerschaftlichem Engagement bleibt da nur die Rolle des "Mitredens « und " Mitmachens «. "Mitentscheiden « ist nicht angesagt, außer bestenfalls in einem zufälligen Gleichklang der Interessenlagen. Dass das von vielen Engagierten häufig als un-

\begin{tabular}{|l|l|} 
Dr. Stefan Nährlich (44) \\
$\begin{array}{l}\text { hat nach einer Berufsausbildung Wirtschaftswissen- } \\
\text { schaften an der Universität Kassel studiert. Er pro- } \\
\text { movierte } 1997 \text { über betriebswirtschaftliche Refor- } \\
\text { men in gemeinnützigen Organisationen. Seit 1999 } \\
\text { leitet er die Geschäfte der Aktiven Bürgerschaft, dem } \\
\text { Kompetenzzentrum für Bürgerengagement der } \\
\text { Volksbanken und Raiffeisenbanken. Deutschland- } \\
\text { weit engagiert sich die Unternehmensgruppe als Cor- } \\
\text { porate Citizen für Bürgerstiftungen. Unterstützt von } \\
\text { Persönlichkeiten aus Gesellschaft, Wirtschaft und } \\
\text { Politik, Wissenschaft und Medien setzt sich die Akti- } \\
\text { ve Bürgerschaft für eine Gesellschaft aktiver Bürger } \\
\text { und engagierter Unternehmen ein. Nährlich hat ver- }\end{array}$ \\
$\begin{array}{l}\text { Münster. } \\
\text { schiedene Lehraufträge, u. a. für »Organisation und Corporate Governance« im Stu- } \\
\text { diengang »Master of Nonprofit-Management and Governance« an der Universität } \\
\text { Internet http://www.aktive-buergerschaft.de }\end{array}$
\end{tabular}

Der Schlüssel zur Macht liegt aber in der Übernahme von Verantwortung. Der Weg zur Verantwortung geht in erster Linie über das "Selbermachen «, weniger über das »Mitmachen «. Wie exotisch der Gedanke offenbar anmutet, konnte man beim Hauptstadtkongress erleben. Als aus dem Publikum danach gefragt wurde, wo denn die Bürgergesellschaft war, als über die Zukunft der Berliner Opernhäuser debattiert wurde, war dies außer mildem Lächeln reihum und auf dem Podium keine weitere Reaktion wert. Dabei ist der Hinweis genau richtig. Der Vorschlag ist konkret, zielgerichtet und beschäftigt sich mit der Lösung eines akuten Berliner Problems. Er knüpft zudem an alte Traditionen an, denn die Deutsche Oper wurde seinerzeit von Charlottenburger Bürgern als Gegengewicht zur königlichen Hofoper Unter den Linden (heute Staatsoper) gegründet. Warum keine Renaissance dieses Konzeptes als private Stiftung oder neuerdings auch möglich - als Genossenschaft? Nicht in Form einer öffentlich-rechtlichen Opernstiftung, sondern in bürgergesellschaftlicher Verantwortung unter Mitfinanzierung aus (unseren) Steuergeldern.

Solche Leuchtturmprojekte wie die Reprivatisierung der Deutschen Oper wären nicht nur wichtige praxisrelevante Schritte mit hoher Symbolkraft, sondern gingen auch konzeptionell in die richtige Richtung und würden deutlich machen, was Bürgergesellschaft im Kern bedeutet: Privat vor Staat. 